\title{
Almost Everywhere Conditions for Hybrid Lipschitz Lyapunov Functions
}

Matteo Della Rossa
Rafal Goebel
Aneel Tanwani

\author{
Luca Zaccarian
}

\begin{abstract}
We introduce a class of locally Lipschitz continuous functions to establish stability of hybrid dynamical systems. Under certain regularity assumptions on system dynamics, we provide sufficient conditions for asymptotic stability on the candidate Lyapunov function. In contrast to the existing literature, these conditions need to be checked only on a dense set using the conventional gradient of certain functions, without the necessity of relying on Clarke's generalized gradient. We discuss the relevance of the stated assumptions with the help of some counterexamples, underlining the subtlety of the proposed relaxation. As an application of our result, we study the stability of a classical example from the reset control literature: the Clegg integrator model, with convex and nonconvex Lyapunov functions, which are almost everywhere differentiable.
\end{abstract}

\section{INTRODUCTION}

Hybrid dynamical systems combine continuous-time and discrete-time evolutions in a unified model, thereby increasing the modeling power of mere differential or difference equations [10]. Lyapunov tools for stability analysis of closed or compact sets with hybrid dynamics have been the subject of extensive research in the past two decades and arguably the most general framework for their representation is well summarized in [8].

Under the so-called hybrid basic conditions characterized in [8], the existence of smooth hybrid Lyapunov functions is necessary and sufficient for asymptotic stability of a compact set. Nevertheless, it often happens that a nonsmooth function $V$ may be easier to describe and construct. Therefore, some relevant nonsmooth results have appeared in [18] and also the follow-up formulations in [14], [20]. In these works, the authors mostly make use of Clarke's generalized gradient to suitably characterize the necessary flow condition at points where $V$ is not differentiable. Among other examples, Clarke's generalized gradient is used in [2], considering piecewise affine Lyapunov functions for state-dependent switching systems and in [13] in the context of interconnected hybrid systems. This strategy, effective for classical continuous-time nonlinear systems, was suggested in [5, Chapter 4] and then well summarized in [21, p. 99]. The main drawback of Clarke's generalized gradient condition is that it requires to look at all the scalar products between generalized gradients and vector fields of the differential inclusion. Less restrictive conditions for stability of differential inclusions are presented in [1], under some regularity assumptions on the (nonsmooth) Lyapunov function. A slightly different

M. Della Rossa, A. Tanwani and L. Zaccarian are with LAAS-CNRS, University of Toulouse (31400), France. R. Goebel is with Department of Mathematics and Statistics, Loyola University Chicago, IL, USA. Corresponding author: mdellaro@laas.fr

Work supported in part by ANR grants CONVAN (number ANR-17CE40-0019-01) and HANDY (number ANR-18-CE40-0010). approach is presented in [9], where the nonsmooth function is studied exploiting regularization-via-convolution technique.

One possible way to overcome the limitations of generalized gradients is to propose stability conditions which only need to be checked away from the points where the candidate Lyapunov function is not differentiable. One main obstruction with this technique is that, since hybrid solutions are constrained to flow in a subset of the state space, it is not possible to directly conclude properties of the directional derivative from "almost everywhere" type of conditions. Furthermore, for hybrid systems, imposing "almost everywhere" conditions on $\nabla V$ is indeed problematic whenever $V$ is not differentiable at the boundary of the so-called "flow set". Indeed, one cannot build a neighborhood around those degenerate points and exploit the fact that $\nabla V$ is well behaved in that neighborhood. This fact has been partially discussed, e.g. in [14, Remark 8, pg. 2575] when discussing the Lyapunov conditions of [15] for reset control systems.

In this paper, we provide a careful treatment of flow conditions on nonsmooth Lyapunov functions for hybrid dynamics that need to be checked only on a dense set. A set of sufficient (and reasonable) conditions is presented, under which it is possible to prove stability of a closed attractor. The class of nonsmooth Lyapunov functions that we consider include those that can be built using pointwise maximum and pointwise minimum of continuously differentiable functions (see [6] and references therein) but are also fairly more general. For illustration purposes, we discuss the relevance of the stated assumptions on the hybrid dynamics, by providing defective examples not satisfying those assumptions where asymptotic stability does not hold even though the "almost everywhere" conditions hold for some $V$. The presented results allow revisiting a classical example from the reset control literature: the Clegg integrator in feedback with an integrating plant. This example has been shown to overcome intrinsic limitations of linear feedback systems in [3]. For this example, both [22] and [14] provided numerical and analytic nonconvex (and nonsmooth) Lyapunov functions. We give here a pair of new (arguably simpler) nonconvex functions, and also a new convex Lyapunov function.

Notation: A function $\alpha: \mathbb{R}_{\geq 0} \rightarrow \mathbb{R}$ is positive definite $(\alpha \in \mathcal{P D})$ if it is continuous, $\alpha(0)=0$, and $\alpha(s)>0$ if $s \neq 0$. A function $\alpha: \mathbb{R}_{\geq 0} \rightarrow \mathbb{R}_{\geq 0}$ is class $\mathcal{K}(\alpha \in \mathcal{K})$ if it is continuous, $\alpha(0)=0$, and strictly increasing. It is said to be class $\mathcal{K}_{\infty}$ if, in addition, it is unbounded. Given $\mathcal{A} \subset \mathbb{R}^{n}$ we denote the distance from $x$ to $\mathcal{A}$ as $|x|_{\mathcal{A}}:=\inf _{y \in \mathcal{A}}|y-x|$, the boundary of $\mathcal{A}$ as $\operatorname{bd}(\mathcal{A})$, the closure as $\overline{\mathcal{A}}$, the interior as $\operatorname{int}(\mathcal{A})$. Given $x \in \mathbb{R}^{n}$, we write $x=\left(x_{1}, \ldots, x_{n}\right)=$ $\left[x_{1}, \ldots, x_{n}\right]^{\top}$. 


\section{Setting And Main Result}

Given closed sets $\mathcal{C}, \mathcal{D} \subset \mathbb{R}^{n}$ and set-valued maps $F$ : $\operatorname{dom} F \rightrightarrows \mathbb{R}^{n}, G: \operatorname{dom} G \rightrightarrows \mathbb{R}^{n}$, such that $\mathcal{C} \subset \operatorname{dom} F$ and $\mathcal{D} \subset \operatorname{dom} G$, consider the hybrid system $\mathcal{H}=(\mathcal{C}, \mathcal{D}, G, F)$, that is

$$
\mathcal{H}: \begin{cases}\dot{x} \in F(x), & x \in \mathcal{C}, \\ x^{+} \in G(x), & x \in \mathcal{D} .\end{cases}
$$

See [8] for a discussion of the hybrid solutions concept, the problem of existence and completeness. A closed set $\mathcal{A} \subset$ $\mathbb{R}^{n}$ is said to be uniformly globally pre-asymptotically stable $(U G p A S)$ for (1) if it is uniformly globally stable, that is

There exists an $\alpha \in \mathcal{K}_{\infty}$ such that any solution $\psi$ of (1) satisfies $|\psi(t, j)|_{\mathcal{A}} \leq \alpha\left(|\psi(0,0)|_{\mathcal{A}}\right)$ for all $(t, j) \in \operatorname{dom} \psi$; and uniformly globally pre-attractive, that is

For each $\varepsilon>0$ and $r>0$ there exists a $T>0$ such that for any solution $\psi$ of (1) with $|\psi(0,0)|_{\mathcal{A}} \leq r$,

$$
\text { if }(t, j) \in \operatorname{dom} \psi \text { and } t+j \geq T \text { then }|\psi(t, j)|_{\mathcal{A}} \leq \varepsilon \text {. }
$$

The prefix "pre-" underlines the fact that the solutions of (1) are not necessary forward complete. In this article, we want to provide sufficient Lyapunov conditions using nonsmooth (but locally Lipschitz) functions. We are interested in relaxing the conditions on the flow set $\mathcal{C}$, thus we introduce here the class of functions that we will consider as Lyapunovcandidate functions.

Definition 1 (The class $\mathcal{L}_{F}(\mathcal{A} ; \mathcal{C})$ ). Let us consider $\mathcal{C} \subset \mathbb{R}^{n}$, a closed set $\mathcal{A} \subset \mathbb{R}^{n}$ and a map $F: \mathcal{C} \rightrightarrows \mathbb{R}^{n}$. A function $V: \operatorname{dom} V \rightarrow \mathbb{R}$ is a locally Lipschitz and locally finitely generated continuous-time Lyapunov function for $\mathcal{A}$ on $\mathcal{C}$, $\left(V \in \mathcal{L}_{F}(\mathcal{A} ; \mathcal{C})\right)$, if

(a) $\overline{\mathcal{C}} \subset \operatorname{dom} V$ and $V$ is locally Lipschitz on $\mathcal{C}$;

(b) there exist $\alpha_{1}, \alpha_{2} \in \mathcal{K}_{\infty}$ such that $\alpha_{1}\left(|x|_{\mathcal{A}}\right) \leq V(x) \leq$ $\alpha_{2}\left(|x|_{\mathcal{A}}\right)$ for every $x \in \mathcal{C}$;

(c) there exists $\rho \in \mathcal{P D}$ and, for every $x \in \mathcal{C}$, there exist an open neighborhood $U \subset \mathbb{R}^{n}$ of $x$, a set $S \subset \mathcal{C}$, a finite index set $I$, and for each $i \in I$, there exist open $U_{i} \subset \mathbb{R}^{n}$ and $\mathcal{C}^{1}$ functions $V_{i}: U_{i} \rightarrow \mathbb{R}$ such that

(i) $\bar{S} \supset \mathcal{C} \cap U$ (i.e., $S$ is dense in $\mathcal{C} \cap U$ ),

(ii) for every $y \in S \cap U$ there exists $i \in I$ such that $y \in$ $U_{i}, V(y)=V_{i}(y), V$ is differentiable at $y, \nabla V(y)=$ $\nabla V_{i}(y)$, and

$$
\left\langle\nabla V_{i}(y), f\right\rangle \leq-\rho\left(|y|_{\mathcal{A}}\right) \quad \forall f \in F(y) .
$$

We will prove that if $V$ is in $\mathcal{L}_{F}(\mathcal{A} ; \mathcal{C})$ then the value of $V$ decreases along the flowing solutions of the hybrid system (1). Collecting this property with positive definiteness and decrease property on the jump set $\mathcal{D}$, we obtain the following Lyapunov theorem for hybrid system (1).

Theorem 1. Given the hybrid system (1), let us suppose that $F: \operatorname{dom} F \rightrightarrows \mathbb{R}^{m}$ is locally bounded and inner semicontinuous ${ }^{1}$. Given a closed set $\mathcal{A}$, suppose that $V$ :

\footnotetext{
${ }^{1}$ A map $F: \mathbb{R}^{n} \rightrightarrows \mathbb{R}^{n}$ is inner semicontinuous at $x$ if $F(x) \subset$ $\liminf _{y \rightarrow x} F(y)=\left\{f \mid \forall x_{k} \rightarrow x, \exists f_{k} \rightarrow f\right.$, with $\left.f_{k} \in F\left(x_{k}\right)\right\}$. The map $F$ is inner semicontinuous if it is inner semicontinuous at each $x \in \mathbb{R}^{n}$.
}

$\operatorname{dom} V \rightarrow \mathbb{R}$ is such that

(a) $V \in \mathcal{L}_{F}(\mathcal{A} ; \mathcal{C})$

(b) $V$ is a discrete-time Lyapunov function of $\mathcal{A}$ on $\mathcal{D}$, in the sense that

(b1) $\operatorname{dom} V \supset \mathcal{D} \cup G(\mathcal{D})$ and there exist $\alpha_{1}, \alpha_{2} \in \mathcal{K}_{\infty}$ such that $\alpha_{1}\left(|x|_{\mathcal{A}}\right) \leq V(x) \leq \alpha_{2}\left(|x|_{\mathcal{A}}\right)$ for every $x \in \mathcal{D} \cup G(\mathcal{D})$;

(b2) there exists $\rho \in \mathcal{P} \mathcal{D}$ such that

$$
V(g)-V(x) \leq-\rho\left(|x|_{\mathcal{A}}\right) \quad \forall x \in \mathcal{D}, g \in G(x) .
$$

Then $\mathcal{A}$ is UGpAS for the hybrid system (1).

We refer to [17, Chapter 5] for the definitions of locally boundedness and a thorough discussion of continuity concepts for set-valued maps.

Remark 1 . We note that there is no loss of generality in using the same class $\mathcal{K}_{\infty}$ functions $\alpha_{1}, \alpha_{2}$ for positive definiteness in $\mathcal{C}$ and in $\mathcal{D} \cup G(\mathcal{D})$ : if the functions were different we could consider the point-wise minimum for the lower bound and the point-wise maximum for the upper bound. The same reasoning applies for the $\mathcal{P} \mathcal{D}$ function $\rho$. More precisely, supposing that (b) of Definition 1 and (b1) of Theorem 1 hold, there must exist $\alpha_{1}, \alpha_{2} \in \mathcal{K}_{\infty}$ such that

$$
\alpha_{1}\left(|x|_{\mathcal{A}}\right) \leq V(x) \leq \alpha_{2}\left(|x|_{\mathcal{A}}\right) \quad \forall x \in \mathcal{C} \cup \mathcal{D} \cup G(\mathcal{D}) .
$$

For the case when $\mathcal{A}$ is compact, it is customary to relax this condition to the equivalent property that $V$ be positive definite w.r.t. $\mathcal{A}$, and radially unbounded relative to $\mathcal{C} \cup \mathcal{D}$, see [11, Lemma 4.3]. Similarly, one can show that (3) is equivalent to requiring that $G(\mathcal{D} \cap \mathcal{A}) \subset \mathcal{A}$ and

$$
V(g)-V(x)<0, \forall x \in \mathcal{D} \backslash \mathcal{A}, \forall g \in G(x),
$$

so that there is no need to compute explicitly the function $\rho$, see [20].

Before proving Theorem 1, we need the following Definition and a preliminary Lemma.

Definition 2. Given system (1), a flowing solution is an absolutely continuous function $\phi:\left[0, T_{\phi}\right) \rightarrow \mathbb{R}^{n}$, with $T_{\phi}>$ 0 , such that

$$
\begin{aligned}
& \phi(t) \in \mathcal{C}, \quad \text { for all } t \in\left[0, T_{\phi}\right), \\
& \dot{\phi}(t) \in F(\phi(t)), \text { almost everywhere in }\left[0, T_{\phi}\right) .
\end{aligned}
$$

We denote the set of all flowing solutions of (1) by $\mathcal{S}_{F}(\mathcal{C})$.

Lemma 1. If $V \in \mathcal{L}_{F}(\mathcal{A} ; \mathcal{C})$ and $F$ is locally bounded and inner semicontinuous, then for every $\phi \in \mathcal{S}_{F}(\mathcal{C})$ and almost every $t \in\left[0, T_{\phi}\right)$,

$$
\frac{d}{d t} V(\phi(\cdot))(t) \leq-\rho\left(|\phi(t)|_{A}\right) .
$$

Proof. Take $\phi \in \mathcal{S}_{F}(\mathcal{C})$, by definition $\phi$ is absolutely continuous, and so is $t \mapsto V(\phi(t))$. Then $\phi$ and $V(\phi(t))$ are differentiable almost everywhere, i.e., for almost every $t \in\left[0, T_{\phi}\right), \dot{\phi}(t)$ and $\frac{d}{d t} V(\phi(\cdot))(t)$ exist, and for almost every such $t, \dot{\phi}(t) \in F(\phi(t))$. Thus consider any such time $t \in\left[0, T_{\phi}\right)$, and denote $x:=\phi(t) \in \mathcal{C}$ and $f:=\dot{\phi}(t) \in F(x)$. 
Let us consider $U$ neighborhood of $x$ given by Definition 1 , the associated index set $I$, the sets $U_{i}$ and functions $\left\{V_{i}\right\}_{i \in I_{U}}$. Let us consider a sequence of times $t_{k} \searrow t$, and denote $x_{k}:=\phi\left(t_{k}\right) \in \mathcal{C}$; without loss of generality we can suppose $x_{k} \in U \cap \mathcal{C}, \forall k \in \mathbb{N}$. Let us consider the set $S$ given by Definition 1; for each $k \in \mathbb{N}$ there exists a subsequence $x_{k, l} \in S \cap U$ converging to $x_{k}$ such that, for each $l \in \mathbb{N}$, there exists $i_{k, l} \in I$ satisfying $V\left(x_{k, l}\right)=V_{i_{k, l}}\left(x_{k, l}\right)$ and $\nabla V\left(x_{k, l}\right)=\nabla V_{i_{k, l}}\left(x_{k, l}\right)$. Since $I$ is finite, one can pass to a subsequence of $x_{k, l}$, without relabeling, so that there exists $i_{k} \in I$ such that, for every $l, i_{k, l}=i_{k}$. Similarly, one can pass to a subsequence of $x_{k}$ so that there exists $i \in I$ such that, for every $k, i_{k}=i$. Then $V\left(x_{k, l}\right)=V_{i}\left(x_{k, l}\right)$, and, by continuity of $V$ and $V_{i}$, letting $l \rightarrow \infty$ yields $V\left(x_{k}\right)=$ $V_{i}\left(x_{k}\right)$ and then letting $k \rightarrow \infty$ yields $V(x)=V_{i}(x)$. Hence

$$
\begin{aligned}
\frac{d}{d t} V(\phi(\cdot))(t) & =\lim _{\tau \searrow t} \frac{V(\phi(\tau))-V(\phi(t))}{\tau-t} \\
& =\lim _{k \rightarrow \infty} \frac{V\left(x_{k}\right)-V(x)}{t_{k}-t} \\
& =\lim _{k \rightarrow \infty} \frac{V_{i}\left(x_{k}\right)-V_{i}(x)}{t_{k}-t} \\
& =\lim _{\tau \searrow t} \frac{V_{i}(\phi(\tau))-V_{i}(\phi(t))}{\tau-t} \\
& =\frac{d}{d t} V_{i}(\phi(\cdot))(t)=\left\langle\nabla V_{i}(x), \dot{\phi}(t)\right\rangle \\
& =\left\langle\nabla V_{i}(x), f\right\rangle .
\end{aligned}
$$

Now, for each $k$, we can choose large enough $l=l_{k}$ so that $x_{k, l_{k}} \rightarrow x$ as $k \rightarrow \infty$. By inner semicontinuity of $F$, we can consider a sequence $f_{k} \in F\left(x_{k, l_{k}}\right)$ such that $f_{k} \rightarrow f$ as $k \rightarrow \infty$. Finally, by continuity, as $k \rightarrow \infty$ we have

$$
\begin{aligned}
(2) \Rightarrow\left\langle\nabla V_{i}\left(x_{k, l_{k}}\right), f_{k}\right\rangle & \leq-\rho\left(\left|x_{k, l_{k}}\right|_{\mathcal{A}}\right), \\
\downarrow & \downarrow \\
\left\langle\nabla V_{i}(x), f\right\rangle & \leq-\rho\left(|x|_{\mathcal{A}}\right),
\end{aligned}
$$

and we can conclude from (7).

We can now prove Theorem 1.

Proof of Theorem 1. Considering a flowing solution $\phi \in$ $\mathcal{S}_{F}(\mathcal{C})$, by using the fundamental theorem of calculus to the function $V \circ \phi$ on $\left[0, T_{\phi}\right)$, we can follow the same steps as in the proof of [8, Theorem 3.18] to get bounds on the flowing solutions between jumps. Condition (3) guarantees decrease in the value of $V \circ \psi$ at all jump times of an hybrid solution $\psi$ of (1).

Remark 2. In [4, Definition 1] and in [19, Chapter 4], the concept of piecewise $\mathcal{C}^{1}$ functions is introduced; this class of functions intuitively describes the set of locally Lipschitz functions obtained by "gluing together" a finite number of continuously differentiable functions. More precise comparisons between locally Lipschitz and locally finitely generated continuous-time Lyapunov functions and the class of piecewise $\mathcal{C}^{1}$ functions are still being investigated.

\section{A Special CASE And Further Discussions}

We now present a special class of locally Lipschitz functions and show that it is computationally easier to check the conditions of Definition 1 for such functions.

Definition 3 (Properly Piecewise $\mathcal{C}^{1}$ functions). Let $V$ : $\operatorname{dom} V \rightarrow \mathbb{R}$ be a continuous function, such that $\mathcal{C} \subset \operatorname{dom} V$. Given $I=\{1, \ldots, K\}$, let us suppose that there exist $\left\{\mathcal{X}_{i}\right\}_{i \in I}$ measurable sets, $\left\{\mathcal{O}_{i}\right\}_{i \in I}$ open sets and continuously differentiable functions $V_{i}: \mathcal{O}_{i} \rightarrow \mathbb{R}, i \in I$, such that:

1) $\mathcal{X}_{i} \cap \mathcal{C} \subset \mathcal{O}_{i}$, for all $i \in I$,

2) $\overline{\operatorname{int}\left(\mathcal{X}_{i}\right)}=\mathcal{X}_{i}$, for all $i \in I$,

3) $\mathcal{X}_{i} \cap \mathcal{X}_{j}=\operatorname{bd}\left(\mathcal{X}_{i}\right) \cap \operatorname{bd}\left(\mathcal{X}_{j}\right)$, for all $i \neq j$,

4) $\mathcal{C} \subset \bigcup_{i \in I} \mathcal{X}_{i}$

5) $V(x)=V_{i}(x)$, if $x \in \mathcal{X}_{i}$.

Then the function $V: \mathcal{C} \cup \mathcal{D} \rightarrow \mathbb{R}$ is called a properly piecewise $\mathcal{C}^{1}$ function on $\mathcal{C}$.

Remark 3. This definition is closely related to [4, Definition 1] recalled in Remark 2. We have added conditions 2) and 3 ) to ensure that the $\mathcal{X}_{i}$ sets are closed, have full measure, and their mutual intersections have measure zero. The same class of functions is studied in [7], in the context of statedependent switched systems.

Proposition 1. Let $V: \mathbb{R}^{n} \rightarrow \mathbb{R}$ be a properly piecewise $\mathcal{C}^{1}$ function on $\mathcal{C} \subset \mathbb{R}^{n}$ such that $\overline{\operatorname{int}(\mathcal{C})}=\mathcal{C}$. Let us consider a closed set $\mathcal{A} \subset \mathbb{R}^{n}$ and a set valued map $F: \operatorname{dom} F \rightrightarrows \mathbb{R}^{n}$ $(\mathcal{C} \subset \operatorname{dom} F)$. If there exist $\alpha_{1}, \alpha_{2} \in \mathcal{K}_{\infty}$, and a $\rho \in \mathcal{P D}$ such that

$$
\begin{gathered}
\alpha_{1}\left(|x|_{\mathcal{A}}\right) \leq V(x) \leq \alpha_{2}\left(|x|_{\mathcal{A}}\right) \quad \forall x \in \mathcal{C} \\
\left\langle\nabla V_{i}(x), f\right\rangle \leq-\rho\left(|x|_{\mathcal{A}}\right), \quad \forall x \in \operatorname{int}\left(\mathcal{X}_{i}\right) \cap \mathcal{C}, \\
\forall f \in F(x), \forall i \in I,
\end{gathered}
$$

then $V \in \mathcal{L}_{F}(\mathcal{A} ; \mathcal{C})$.

Proof. The facts that $\overline{\mathcal{C}} \subset \operatorname{dom} V$ and that $V$ is locally Lipschitz on $\mathcal{C}$ are straightforward. Condition (8) is exactly (b) of Definition 1. For proving (c), let us consider a point $x \in \mathcal{C}$ and a neighborhood $U$ of $x$. Let us define $S:=\bigcup_{i \in I} \operatorname{int}\left(\mathcal{X}_{i}\right) \cap \mathcal{C}$; noting that condition 2) implies $\operatorname{meas}\left(\operatorname{bd}\left(\mathcal{X}_{i}\right)\right)=0$, (where meas is the Lebesgue measure) and recalling that the complement of a negligible set is dense, we have $\bar{S} \supset \mathcal{C} \cap U$. De to to conditions 3) and 5) of Definition 3, for each $y \in S$ there exists a unique $i \in I$ such that $V(y)=V_{i}(y)$ and $\nabla V(y)=\nabla V_{i}(y)$. The conclusion follows from (9).

We have shown that for a properly piecewise $\mathcal{C}^{1}$ function, we have a simple way to verify condition (2): it suffices to verify the Lyapunov inequality

$$
\langle\nabla V(x), f\rangle \leq-\rho\left(|x|_{\mathcal{A}}\right),
$$

almost everywhere in $\mathcal{C}$, that is in the $\operatorname{set} \mathcal{C} \cap \bigcup_{i \in I} \operatorname{int}\left(\mathcal{X}_{i}\right)$. It is interesting to note that

$$
\mathcal{N}_{V} \subset \bigcup_{i \in I} \operatorname{bd}\left(\mathcal{X}_{i}\right),
$$




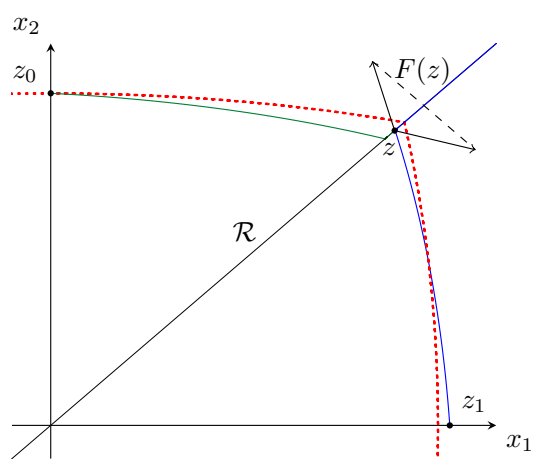

Fig. 1: Counterexample 1: In red dashed line a level set of $V$ in (13), in green and blue the trajectories of two solutions. The solution starting from $z_{1}$ enters the plotted sublevel set, reaches the point $z \in \mathcal{R}$ and then starts "sliding".

where $\mathcal{N}_{V}:=\left\{x \in \mathbb{R}^{n} \mid \nabla V(x)\right.$ is not defined $\}$ by the Rademacher Theorem, has measure zero.

Remark 4 (Max and Min Functions). A common subclass of properly piecewise $\mathcal{C}^{1}$ functions is obtained by applying the $\max$ and $\min$ operators to a finite collection of continuously differentiable functions. More precisely, given a family $\mathcal{V}=\left\{V_{1}, \ldots, V_{K}\right\} \subset \mathcal{C}^{1}(\mathcal{O}, \mathbb{R})$, where $\mathcal{O}$ is an open set containing $\mathcal{C} \cup \mathcal{D}$, the functions

$$
V_{M}(x):=\max _{i \in I} V_{i}(x) \text { and } V_{m}(x):=\min _{i \in I} V_{i}(x)
$$

satisfy the hypothesis of Definition 3 [4, Theorem 1(b)]. Taking $V_{M}$ as an example, we can also characterize the sets $\mathcal{X}_{i}$ as follows

$$
\mathcal{X}_{i}=\overline{\left\{x \in \mathbb{R}^{n} \mid V_{i}(x)>V_{j}(x), \forall j \in I, j \neq i\right\}} .
$$

In the following counterexample, we show that the inner semicontinuity of $F: \mathbb{R}^{n} \rightrightarrows \mathbb{R}^{n}$ is crucial in our result.

Counterexample 1 (Violating Inner Semicontinuity). Consider the state-dependent switching system defined by $\mathcal{C}=$ $\mathbb{R}^{2}, \mathcal{D}=G=\emptyset$ and $F: \mathbb{R}^{2} \rightrightarrows \mathbb{R}^{2}$ is the Filippov regularization of the following discontinuous linear system:

$$
\dot{x}= \begin{cases}A_{1} x, & \text { if } x^{\top} Q x \geq 0, \\ A_{2} x, & \text { if } x^{\top} Q x<0\end{cases}
$$

where

$A_{1}=\left[\begin{array}{cc}-0.3 & -1 \\ 5 & -0.3\end{array}\right], A_{2}=\left[\begin{array}{cc}-0.3 & 5 \\ -1 & -0.3\end{array}\right], Q=\left[\begin{array}{cc}1 & 0 \\ 0 & -1\end{array}\right]$.

By definition of Filippov regularization we have

$$
F(x)= \begin{cases}\left\{A_{1} x\right\}, & \text { if } x^{\top} Q x>0 \\ \left\{A_{2} x\right\}, & \text { if } x^{\top} Q x<0 \\ \overline{\operatorname{co}}\left\{A_{1} x, A_{2} x\right\} & \text { if } x^{\top} Q x=0\end{cases}
$$

It is easy to see that $F$ is outer semicontinuous with compact and convex values, but it is not inner semicontinuous. Let us consider the candidate Lyapunov function

$$
V(x)=\max \left\{x^{\top} P_{1} x, x^{\top} P_{2} x\right\},
$$

where $P_{1}=\left[\begin{array}{ll}5 & 0 \\ 0 & 1\end{array}\right]$ and $P_{2}=\left[\begin{array}{ll}1 & 0 \\ 0 & 5\end{array}\right]$. By Remark 1 this function satisfies (4) because $P_{1}>0$ and $P_{2}>0$, and (3) holds vacuously because $\mathcal{D}=\emptyset$. It is easy to check that

$$
\begin{aligned}
& \text { - } P_{1} A_{1}+A_{1}^{\top} P_{1}<0, \\
& \text { - } P_{2} A_{2}+A_{2}^{\top} P_{2}<0, \\
& \text { - } x^{\top} Q x>0 \Leftrightarrow x^{\top} P_{1} x>x^{\top} P_{2} x, \\
& \text { - } \operatorname{bd}\left(\mathcal{X}_{1}\right) \cup \operatorname{bd}\left(\mathcal{X}_{2}\right)=\left\{x \in \mathbb{R}^{2} \mid x^{\top} Q x=0\right\} .
\end{aligned}
$$

where the set $\mathcal{X}_{i}$ are defined as in Remark 4. That means $\langle\nabla V(x), f\rangle<0 ; \forall x \in \mathbb{R}^{2} \backslash \operatorname{bd}\left(\mathcal{X}_{1}\right) \cup \operatorname{bd}\left(\mathcal{X}_{2}\right), \forall f \in F(x)$, which implies (9). On the other hand, one can see that every flowing solution starting at some $x_{0} \in \mathcal{R}:=\left\{x \in \mathbb{R}^{2} \mid x_{1}=\right.$ $\left.x_{2}\right\}, x_{0} \neq 0$ goes to infinity sliding along $\mathcal{R}$. See Figure 1 for a graphical representation.

\section{A Case Study: Clegg integrator}

The Clegg integrator connected to an integrating plant has been shown to overcome intrinsic limitations of linear feedback, [3], (see also [16]). More specifically, using the $\varepsilon$-regularization suggested in [14], we focus on the closedloop, considering the hybrid system

$$
\begin{cases}\dot{x}=A_{F} x, & x \in \mathcal{C}=\left\{x \in \mathbb{R}^{2} \mid x^{\top} Q x \geq 0\right\}, \\ x^{+}=A_{J} x, & x \in \mathcal{D}=\left\{x \in \mathbb{R}^{2} \mid x^{\top} Q x \leq 0\right\},\end{cases}
$$

with

$$
A_{F}=\left[\begin{array}{cc}
0 & 1 \\
-1 & 0
\end{array}\right], A_{J}=\left[\begin{array}{ll}
1 & 0 \\
0 & 0
\end{array}\right], Q=\left[\begin{array}{cc}
1 & -\frac{1}{2 \varepsilon} \\
-\frac{1}{2 \varepsilon} & 0
\end{array}\right],
$$

and $\varepsilon>0$ being a small regularization parameter. Following [22], there does not exist a quadratic Lyapunov function. In fact, given a symmetric and positive definite matrix $P=\left[\begin{array}{ll}p_{11} & p_{12} \\ p_{12} & p_{22}\end{array}\right]$, and considering points $z_{1}=(-1,0)$ and $z_{2}=(0,1)$, we have that $z_{1}, z_{2} \in \mathcal{C}$ and the Lyapunov inequalities $z_{1}^{\top} P A_{F} z_{1}<0$ and $z_{2}^{\top} P A_{F} z_{2}<0$ would imply $p_{12}<0$ and $p_{12}>0$ respectively and hence, a contradiction. UGAS of (14) was established with nonconvex numerical piecewise quadratic constructions in [22], and then via a nonconvex analytic construction in [14]. We illustrate the use of Theorem 1 by building three Lyapunov functions, one of them being convex. In what follows, we fix $\varepsilon=0.1$, but the functions that we construct work for any $\varepsilon$ such that $0<$ $\varepsilon \leq 0.1$. We underline that none of these functions satisfies the "classical" Clarke's generalized gradient conditions, i.e. $\left\langle v, A_{F} x\right\rangle \geq 0$, for some $x \in \operatorname{bd}(\mathcal{C})$ and for some $v$ contained in the generalized gradient of $V$ at $x$. For further details about Clarke's gradient-based conditions we refer to [5] and [21].

\section{A. Max of 2 sign-indefinite quadratics}

We first use a max function of two quadratics

$$
\begin{gathered}
V_{M}(x):=\max \left\{x^{\top} P_{1} x, x^{\top} P_{2} x\right\}, \\
\text { with } P_{1}=\left[\begin{array}{cc}
1 & -0.1 \\
-0.1 & 0.5
\end{array}\right], P_{2}=\left[\begin{array}{ll}
2.5 & 1.4 \\
1.4 & 0.5
\end{array}\right],
\end{gathered}
$$




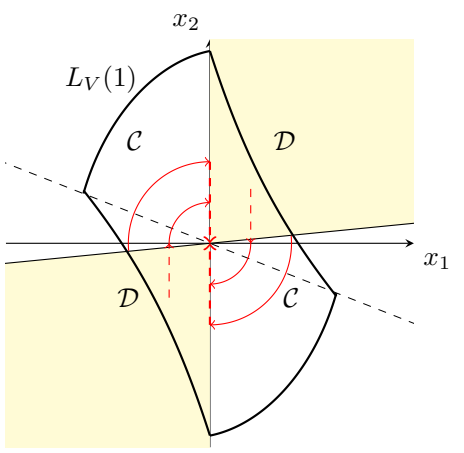

(a) In black we plotted the 1-level set of the max of quadratics Lyapunov function $V_{M}$ defined in (15).

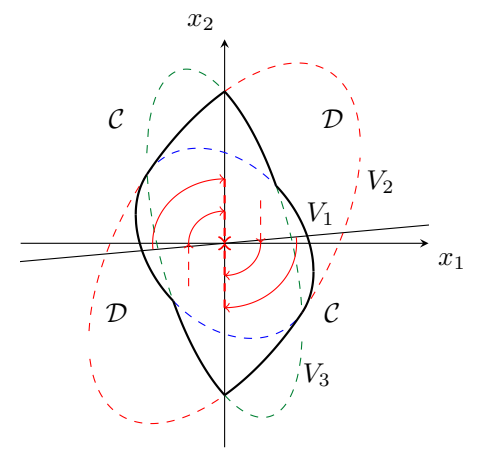

(b) The 1-level set of the mid of quadratics (c) The 1-level set of the convex Lyapunov Lyapunov function $V_{\text {mid }}$ defined in (16).

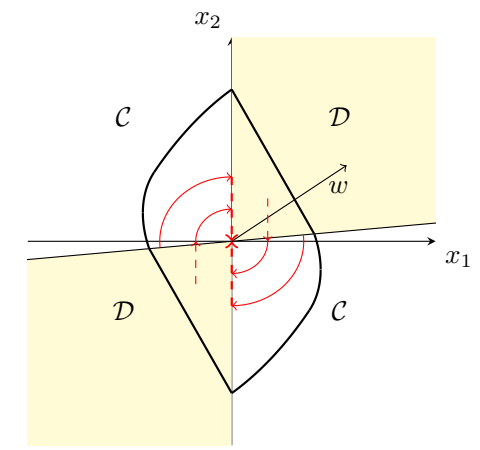

function $V_{\text {conv }}$ defined in (17).

Fig. 2: Level sets of the constructed Lyapunov functions for system (14); in red some particular solutions.

where we illustrate the use of quadratics not necessarily positive definite (see also [12]). We prove next that (15) satisfies the hypotheses of Theorem 1. First of all, the function $V_{M}$ satisfies (4) (positive definiteness): this is straightforward by noting that $P_{1}>0$. Following Remark 4, we characterize here the sets $\mathcal{X}_{1}, \mathcal{X}_{2}$ writing

$$
\begin{aligned}
& \mathcal{X}_{1}:=\left\{x \mid V_{1}(x) \geq V_{2}(x)\right\}=\left\{x \mid x^{\top}\left(P_{1}-P_{2}\right) x \geq 0\right\}, \\
& \mathcal{X}_{2}:=\left\{x \mid V_{2}(x) \geq V_{1}(x)\right\}=\left\{x \mid x^{\top}\left(P_{2}-P_{1}\right) x \geq 0\right\}
\end{aligned}
$$

and denoting $S:=\left(P_{1}-P_{2}\right)=\left[\begin{array}{cc}-1.5 & -1.5 \\ -1.5 & 0\end{array}\right]$, we can conclude

$$
\mathcal{X}_{1}=\left\{x \mid x^{\top} S x \geq 0\right\}, \mathcal{X}_{2}=\left\{x \mid x^{\top} S x \leq 0\right\} .
$$

We also note that $S$ can be decomposed as $S=\theta_{1} \theta_{2}^{\top}+\theta_{2} \theta_{1}^{\top}$, where $\theta_{1}, \theta_{2}$ are vectors perpendicular in directions to the lines where $V_{1}=V_{2}$, that is $\theta_{1}=(1.5,0), \theta_{2}=(-1,-2)$.

For the jump condition (3), we first note that $\mathcal{D} \subset$ $\left\{x \mid x_{1} x_{2} \geq 0\right\} \subset \mathcal{X}_{2}$ : given $x=\left(x_{1}, x_{2}\right)$ with $x_{1} x_{2} \geq 0$ we have $x^{\top} S x \leq 0$. Thus, for each $x=\left(x_{1}, x_{2}\right) \in \mathcal{D} \cup G(\mathcal{D})$, $V(x)=V_{2}(x)$ and we may prove the equivalent property in (5)

$$
\begin{aligned}
V_{2}\left(x^{+}\right)-V_{2}(x) & =x^{\top} A_{J}^{\top} P_{2} A_{J} x-x^{\top} P_{2} x \\
& =2.5 x_{1}^{2}-2.5 x_{1}^{2}-3 x_{1} x_{2}-0.5 x_{2}^{2}<0,
\end{aligned}
$$

$\forall x \in \mathcal{D} \backslash \mathcal{A}$, where we used the fact that $\mathcal{D} \backslash \mathcal{A} \subset\left\{x \mid x_{1} x_{2} \geq\right.$ $\left.0, x_{2} \neq 0\right\}$. In light of Remark 1, we can conclude that (3) holds. For the flow condition, we will show the condition (9) of Proposition 1. We split the flow set $\mathcal{C}=\mathcal{C}_{1} \cup \mathcal{C}_{2}:=$ $\left(\mathcal{X}_{1} \cap \mathcal{C}\right) \cup\left(\mathcal{X}_{2} \cap \mathcal{C}\right)$. From the fact that $\mathcal{D} \subset \mathcal{X}_{2}$, we note that $\left(\mathcal{X}_{1} \cap \mathcal{C}\right)=\mathcal{X}_{1}$. Thus for $\mathcal{C}_{1}$, it suffices to verify that

$$
\left\langle\nabla V_{1}(x), A_{F} x\right\rangle \leq-0.1|x|^{2} \text {, if } x \in \mathcal{X}_{1}=\left\{x \mid x^{\top} S x \geq 0\right\} .
$$

This can be done by finding a $\mu_{1} \geq 0$ such that the LMI

$$
P_{1} A_{F}+A_{F}^{\top} P_{1}+\mu_{1} S+0.1 \mathrm{Id}<0
$$

is satisfied, and this is the case choosing, for example, $\mu_{1}=$ 0.4 . Secondly, we note that the region $\mathcal{C}_{2}:=\mathcal{X}_{2} \cap \mathcal{C}$ can be rewritten as $\mathcal{C}_{2}=\left\{x \mid x^{\top} Q_{2} x \geq 0\right\}$, where

$$
Q_{2}:=\theta_{2} \theta_{3}^{\top}+\theta_{3}^{\top} \theta_{2}=\left[\begin{array}{cc}
0.2 & -0.8 \\
-0.8 & -4
\end{array}\right],
$$

and $\theta_{3}=(-0.1,1)$ is the perpendicular direction of the line $x_{2}=\varepsilon x_{1}=0.1 x_{1}$ that represents the boundary of $\mathcal{C}$ that intersects $\mathcal{X}_{2}$. Thus, for proving

$$
\left\langle\nabla V_{M}(x), A_{F} x\right\rangle<-0.1|x|^{2}, \forall x \in \operatorname{int}\left(\mathcal{X}_{2}\right) \cap \mathcal{C},
$$

it suffices to find a $\mu_{2} \geq 0$ such that the LMI

$$
P_{2} A_{F}+A_{F}^{\top} P_{2}+\mu_{2} Q_{2}+0.1 \mathrm{Id}<0,
$$

is satisfied. This is the case, for example, with $\mu_{2}=2$. To summarize, all the conditions of Theorem 1 are satisfied by $V_{M}$ and we can conclude that $\mathcal{A}=\{0\}$ is UpGAS for system (14). See Fig. 2(a) for a graphical representation of our construction, where nonconvexity of $V_{M}$ emerges from the fact that $P_{2}$ is not sign-definite.

\section{B. Mid of quadratics}

Consider the symmetric matrices

$P_{1}=\left[\begin{array}{cc}1 & 0.25 \\ 0.25 & 0.7\end{array}\right], P_{2}=\left[\begin{array}{cc}0.55 & -0.2 \\ -0.2 & 0.25\end{array}\right], P_{3}=\left[\begin{array}{cc}\frac{25}{16} & \frac{49}{160} \\ \star & 0.25\end{array}\right]$

and consider the function

$$
\begin{aligned}
V_{\text {mid }}(x) & :=\operatorname{mid}\left\{V_{1}(x), V_{2}(x), V_{3}(x)\right\} \\
& :=\max \left\{\min \left\{V_{1}, V_{2}\right\}, \min \left\{V_{2}, V_{3}\right\}, \min \left\{V_{1}, V_{3}\right\}\right\},
\end{aligned}
$$

where $V_{i}(x):=x^{\top} P_{i} x$. Intuitively, the "mid" operator selects the function whose value lies between the two others. Taking $I=\{1,2,3\}$ we introduce the sets in Definition 3 as

$$
\begin{aligned}
\mathcal{X}_{i}: & =\operatorname{cl}\left(\left\{x \in \mathbb{R}^{2} \mid x^{\top} P_{j} x<x^{\top} P_{i} x<x^{\top} P_{k} x\right\}\right. \\
& \left.\cup\left\{x \in \mathbb{R}^{2} \mid x^{\top} P_{k} x<x^{\top} P_{i} x<x^{\top} P_{j} x\right\}\right)
\end{aligned}
$$

for every $i \in I, j \neq k \in I \backslash\{i\}$. It is easy to see that $\left\{\mathcal{X}_{i}\right\}_{i \in I}, \mathcal{O}_{i} \equiv \mathbb{R}^{n},\left\{V_{i}\right\}_{i \in I}$ and $V_{\text {mid }}$ satisfy the hypothesis of Definition 3. Let us now prove the conditions of Theorem 1. Positive definiteness of $V_{\text {mid }}$ is immediate, noting that $P_{1}, P_{2}$ and $P_{3}$ are all positive definite. 
The jump condition on $\mathcal{D}$ is trivial, and can be obtained following the same steps as with function $V_{M}$.

For the flow condition it can be seen that

$$
\begin{aligned}
& \mathcal{X}_{1} \cap \operatorname{int}(\mathcal{C})=\left\{x \mid x^{\top} Q_{1} x>0\right\}, \\
& \mathcal{X}_{2} \cap \operatorname{int}(\mathcal{C})=\left\{x \mid x^{\top} Q_{2} x>0\right\}, \mathcal{X}_{3} \cap \operatorname{int}(\mathcal{C})=\emptyset,
\end{aligned}
$$

where

$$
\begin{aligned}
& Q_{1}=\theta_{1} \theta_{2}^{\top}+\theta_{2} \theta_{1}^{\top}=\left[\begin{array}{cc}
0.2 & -0.9 \\
-0.9 & -2
\end{array}\right], \\
& Q_{2}=\theta_{2} \theta_{3}^{\top}+\theta_{3} \theta_{2}^{\top}=\left[\begin{array}{cc}
-2 & -1 \\
-1 & 0
\end{array}\right] .
\end{aligned}
$$

As in the previous example, $\theta_{1}=(-0.1,1), \theta_{2}=(-1,-1)$ and $\theta_{3}=(1,0)$ are properly chosen vectors perpendicular to the lines that represent the boundaries of $\mathcal{X}_{1} \cap \operatorname{int}(\mathcal{C})$ and $\mathcal{X}_{2} \cap$ $\operatorname{int}(\mathcal{C})$, respectively. As before we prove the flow conditions (9) looking for scalars $\mu_{1}, \mu_{2} \geq 0$ such that the LMIs

$$
\begin{aligned}
& P_{1} A_{F}+A_{F}^{\top} P_{1}+\mu_{1} Q_{1}+0.1 \mathrm{Id}<0, \\
& P_{2} A_{F}+A_{F}^{\top} P_{2}+\mu_{2} Q_{2}+0.1 \mathrm{Id}<0,
\end{aligned}
$$

are satisfied; this turns out to be true choosing, for example, $\mu_{1}=\mu_{2}=0.5$. We have proved that

$$
\begin{aligned}
& \left\langle\nabla V_{1}(x), A_{F} x\right\rangle \leq-0.1|x|^{2}, \forall x \in \mathcal{C}_{1}, \text { and } \\
& \left\langle\nabla V_{2}(x), A_{F} x\right\rangle \leq-0.1|x|^{2}, \forall x \in \mathcal{C}_{2}
\end{aligned}
$$

which imply the flow conditions (9) and thus Proposition 1 holds. All the conditions of Theorem 1 are satisfied and we conclude that $\mathcal{A}=\{0\}$ is UpGAS for the system (14). See Fig. 2(b) for a graphical representation of our construction, which shows again nonconvex level sets of $V_{\text {mid }}$.

\section{Convex Lyapunov Function}

The two Lyapunov functions above are both nonconvex. We construct here a convex one, starting from $V_{\text {mid }}$. Looking at the level set $L_{V_{\text {mid }}}(1)$, the idea is to connect the points of intersection of $L_{V_{\text {mid }}}(1)$ with the two lines that form the boundary of $\mathcal{D}$ using a straight line. We thus define

$$
V_{\text {conv }}(x)= \begin{cases}V_{\text {mid }}(x), & \text { if } x \in \mathcal{C}, \\ \langle w, x\rangle^{2}, & \text { if } x \in \mathcal{D},\end{cases}
$$

where $w=(0.9574,0.7071)$ is a vector tangent to the line of interest, suitably scaled to ensure continuity. This function satisfies the conditions of Theorem 1 from the properties of $V_{\text {mid }}$. It is represented in Fig. 2(c).

\section{CONCLusion}

In the context of analysis of stability of hybrid systems, we introduce a class of locally Lipschitz functions for which the Lyapunov inequality needs to be checked only on a dense set around each point of the flow set $\mathcal{C}$. In the first place this result permits us to look at a class of nonsmooth functions that includes max and min over a finite family of smooth functions. On the other hand the analysis is carried out without considering the Clarke's generalized gradient. We apply our result to a classical example from the reset systems literature: the Clegg integrator model. As further research, the relations between the proposed class of locally Lipschitz functions and the piecewise $\mathcal{C}^{1}$ functions recently proposed in the literature remain to be investigated.

\section{REFERENCES}

[1] A. Bacciotti and F. Ceragioli. Stability and stabilization of discontinuous systems and nonsmooth Lyapunov functions. ESAIM: Control, Optimisation and Calculus of Variations, 4:361-376, 1999.

[2] R. Baier, L. Grüne, and S. F. Hafstein. Linear programming based Lyapunov function computation for differential inclusions. Discrete \& Continuous Dynamical Systems - B, 17:33, 2012.

[3] O. Beker, C. V. Hollot, and Y. Chait. Plant with integrator: An example of reset control overcoming limitations of linear feedback. IEEE Transactions on Automatic Control, 46:1797 - 1799, 122001.

[4] R. Chaney. Piecewise $\mathcal{C}^{k}$ functions in nonsmooth analysis. Nonlinear Analysis: Theory, Methods and Applications, 15(7):649 - 660, 1990.

[5] F. H. Clarke, Y. S. Ledyaev, R. J. Stern, and P. R. Wolenski. Nonsmooth Analysis and Control Theory, volume 178 of Graduate Texts in Mathematics. Springer-Verlag, New York, 1998.

[6] M. Della Rossa, A. Tanwani, and L. Zaccarian. Max-min Lyapunov functions for switching differential inclusions. 57th IEEE-Conference on Decision and Control, pages 5664-5669, 2018.

[7] M. Della Rossa, A. Tanwani, and L. Zaccarian. Smooth approximation of patchy Lyapunov functions for switching systems. In Proc. 11th IFAC Symposium on Nonlinear Control Systems (NolCoS), 2019.

[8] R. Goebel, R. Sanfelice, and A. Teel. Hybrid Dynamical Systems: Modeling, Stability, and Robustness. Princeton University Press, 2012.

[9] M. Grzanek, A. Michalak, and A. Rogowski. A nonsmooth Lyapunov function and stability for ODEs of Caratheodory type. Nonlinear Analysis: Theory, Methods and Applications, 69:337-342, 072008.

[10] T. A. Henzinger. The theory of hybrid automata. In Proceedings 11th Annual IEEE Symposium on Logic in Computer Science, pages 278-292, July 1996.

[11] H. K. Khalil. Nonlinear Systems. Prentice Hall, 2002.

[12] Y. Li, Z. Lin, and N. Li. Stability and performance analysis of saturated systems using an enhanced max quadratic Lyapunov function. 50(1):11847 - 11852, 2017. 20th IFAC World Congress.

[13] D. Liberzon, D. Nešić, and A. R. Teel. Lyapunov-based smallgain theorems for hybrid systems. IEEE Transactions on Automatic Control, 59(6):1395-1410, June 2014.

[14] D. Nešić, A. R. Teel, and L. Zaccarian. Stability and performance of SISO control systems with first-order reset elements. IEEE Transactions on Automatic Control, 56:2567 - 2582, 122011.

[15] D. Nešić, L. Zaccarian, and A. R. Teel. Stability properties of reset systems. Automatica, 44:2019-2026, 2008.

[16] C. Prieur, I. Queinnec, S. Tarbouriech, and L. Zaccarian. Analysis and synthesis of reset control systems. Foundations and Trends in Systems and Control, 6:117-338, 012018.

[17] R. Rockafellar and R.-B. Wets. Variational Analysis, volume 317 of Gundlehren der mathematischen Wissenchaften. Springer-Verlag, Berlin, 3rd printing, 2009 edition, 1998.

[18] R. G. Sanfelice, R. Goebel, and A. R. Teel. Invariance principles for hybrid systems with connections to detectability and asymptotic stability. IEEE Transactions on Automatic Control, 52:2282-2297, 2007.

[19] S. Scholtes. Introduction to Piecewise Differentiable Equations. Springer Briefs in Optimization. Springer-Verlag, New York, 2012.

[20] A. Seuret, C. Prieur, S. Tarbouriech, A. R. Teel, and L. Zaccarian. A nonsmooth hybrid invariance principle applied to robust event-triggered design. IEEE Transactions on Automatic Control, 64(5):2061-2068, 2018.

[21] A. R. Teel and L. Praly. On assigning the derivative of a disturbance attenuation control Lyapunov function. Mathematics of Control, Signals and Systems, 13(2):95-124, 2000.

[22] L. Zaccarian, D. Nešić, and A. R. Teel. Analytical and numerical Lyapunov functions for SISO linear control systems with first-order reset elements. International Journal of Robust and Nonlinear Control, 21(10):1134-1158, 2011. 